\title{
Evaluation de deux méthodes de correction de biais des sorties de modèles climatiques régionaux Cordex-Africa pour la prévision des pluies : cas du bassin côtier oranais
}

\author{
Sabrina Taïbi ${ }^{1}$, Ayoub Zeroual ${ }^{2}$, and Naziha Melhani ${ }^{1}$ \\ ${ }^{1}$ Département des Sciences de l'Eau et l'Environnement, L-PPRE, Université de Blida, Blida, Algérie \\ ${ }^{2}$ École Nationale Supérieure d'Hydraulique, L-GEE, 09000, Blida, Algérie
}

Correspondence: Sabrina Taïbi (taibisabrina86@gmail.com)

Published: 16 November 2021

\begin{abstract}
Résumé. Ce travail vise à évaluer les pluies simulées issues des sorties de modèles climatiques régionaux Cordex-Africa dans le bassin côtier oranais en Algérie. Pour cela les simulations du modèle RCA4 (Rossby Centre Atmosphere model, version 4) forcé par deux modèles de circulation globale (MPI-ESM-LR et CNRMCM5) sous deux scenarios de forçages radiatifs « Representative Concentration Pathways » (RCPs) RCP 4.5 et RCP 8.5 sont comparées aux pluies observées au niveau de cinq stations pluviométriques, au cours de la période de contrôle 1981-2005 à l'échelle mensuelle. Les données futures simulées sont ensuite corrigées à l'aide de deux méthodes de correction de biais, à savoir, la méthode quantile-quantile et la méthode Delta, afin de mieux analyser leur évolution au cours de la période de projection 2075-2099. Les coefficients d'échange estimés au cours de la période 2075-2099 montrent que les simulations corrigées par la méthode Delta sont moins biaisées que les simulations corrigées par la méthode quantile-quantile. L'analyse de l'évolution future des pluies met en évidence une réduction de $-12 \%$ à $-38 \%$ d'ici la fin du 21 ème siècle selon le RCP 4.5. Cette réduction qui est encore plus importante selon le scénario pessimiste RCP 8.5, risque d'affecter la disponibilité des ressources en eau dans la région qui a connu par le passé une période de sècheresse sévère et persistante. Enfin, cette étude peut être utilisée comme outil d'aide à la décision destiné aux parties prenantes de la gestion intégrée des ressources en eau et de l'agriculture. Néanmoins, pour une meilleure appréciation des impacts socio-économiques, une étude plus approfondie en considérant plusieurs modèles climatiques et d'autres paramètres climatiques, est recommandée.
\end{abstract}

\section{Introduction}

Du fait de sa position géographique, l'Algérie fait partie des pays les plus vulnérables aux changements climatiques. Des études ont déjà mis en évidence une augmentation des températures de $0.2-0.4^{\circ} \mathrm{C} /$ décennie (Zeroual et al., 2017) ainsi qu'une une baisse de la pluviométrie particulièrement dans la partie Nord-Ouest de l'Algérie (Meddi et al., 2010; Bekkoussa et al., 2008) caractérisée par un déficit de $13 \%-$ $35 \%$ (Taibi et al., 2013). Ainsi, les potentialités en eaux superficielles du Nord de l'Algérie sont passées de 12,5 milliards de $\mathrm{m}^{3}$ à 10 milliards de $\mathrm{m}^{3}$ (ANRH, 2007).
Les évènements extrêmes (sécheresse, pluviométrie déficitaire, augmentation des températures) ont eu des conséquences néfastes sur la satisfaction des besoins en eau des différents secteurs socio-économiques. Pour le GIEC (2014), cette situation risque de s'exacerber pour les années à venir.

En effet, selon les différents scénarios d'émissions (GIEC, 2014) les modèles climatiques prédisent une augmentation de la température de $1{ }^{\circ} \mathrm{C}$ à $4{ }^{\circ} \mathrm{C}$ d'ici la fin du 21ème siècle. Ceci risque encore d'accentuer les événements extrêmes particulièrement les sécheresses dans le bassin méditerranéen (GIEC, 2014) et affectera sensiblement la disponibilité des 
ressources en eau pour les différents usagers particulièrement l'agriculture, secteur le plus consommateur d'eau.

Face à cette situation, les études d'impact socioéconomique et environnemental des changements climatiques et surtout pour la prévision, nécessitent l'utilisation des données sorties de modèles climatiques régionaux à haute résolution, tel que les modèles développés par le projet CORDEX qui offre des sorties de modèles climatiques pour différentes résolutions $0.44,0.22$ et $0.11^{\circ}$ (https://cordex. org/, la date du dernier accès : 16 juin 2021). Cependant, l'utilisation directe des sorties des modèles régionaux dans les études d'impacts reste relativement limitée du fait qu'elles sont biaisées par rapport aux observations et nécessitent une correction afin d'affiner les simulations (Teutschbein and Seibert, 2012; Ly et al., 2019). Pour y remédier, des méthodes de correction de biais sont utilisées, telles que la méthode Delta et la méthode quantile-quantile (Mandez et al., 2020; Dobler et al., 2008; Fang et al., 2015).

C'est dans ce contexte que s'inscrit ce travail qui porte sur l'évaluation de deux méthodes de correction de biais des sorties de modèles climatiques pour la prévision des pluies dans le bassin côtier oranais en Algérie. Pour cela les simulations du modèle RCA4 (Rossby Centre Atmosphere model, version 4) forcé par deux modèles de circulation globale (MPI-ESM-LR et CNRM-CM5) sous deux scenarios de forçages radiatifs «Representative Concentration Pathways » (RCPs) RCP 4.5 et RCP 8.5 ont été utilisées. Les données futures des pluies simulées sont corrigées par les méthodes quantile-quantile et Delta avant d'être utilisées pour l'analyse de l'évolution des pluies du bassin côtier oranais au cours de la période de projection 2075-2099.

\section{Présentation du secteur d'étude et données utilisées}

Le bassin Côtier Oranais fait partie de la région hydrographique Oranie-Chott Chergui qui se trouve à l'Ouest de l'Algérie. Il est situé au Nord des bassins de la Tafna, la Macta et du Chott Chergui et limité au Nord par la mer Méditerranée.

L'étude a été menée sur cinq stations pluviométriques réparties sur l'ensemble de la zone d'étude (Fig. 1). Les données mensuelles ont été collectées auprès de l'Agence Nationale des Ressources Hydrauliques (ANRH) pour la période d'étude 1981-2010.

La pluie moyenne annuelle de la zone d'étude varie entre $249 \mathrm{~mm}$ à la station d'El braya à $353 \mathrm{~mm}$ à Oued Berkeche (Tableau 1). Effectivement, l'Ouest de l'Algérie est caractérisé par une pluviométrie inférieure à celle du centre et de l'Est du pays. C'est également la partie du pays qui a connu une longue période de sécheresse particulièrement au cours des années 80. Afin d'évaluer les sorties de modèles climatiques régionaux de Cordex-Africa, les modèles RCA4MPI-ESM-LR et RCA4-CNRM-CM5 ont été utilisés. Ces modèles ont été jugés satisfaisants pour l'Algérie par rap- port aux autres modèles disponibles (Zeroual et al., 2019) et offrent des simulations à une résolution de $50 \mathrm{~km}$.

\section{Méthodes}

Pour évaluer la capacité des modèles climatiques à reproduire la variabilité pluviométrique passée du secteur d'étude, les données simulées par les deux MCR sont comparées aux pluies mensuelles observées au niveau de chacune des cinq stations au cours de la période de référence 1981-2005 par l'estimation du biais Eq. (1).

Biais $=\frac{\text { Pluie simulée-pluie observée }}{\text { pluie observée }}$

Il existe différentes méthodes de correction de biais qui permettent de corriger les simulations futures en se basant sur la différence entre les données observées et simulées au cours de la période de contrôle. Toutefois, les méthodes Delta et quantile-quantile sont le plus souvent utilisées (Tong et al., 2020; Mandez et al., 2020). Ces deux méthodes ont été appliquées dans le cadre de ce travail, afin de corriger les simulations futures issues des deux MCR (MPI et CNRM) au cours de la période de projection 2075-2099 et pour les deux scénarios d'émissions RCP 4.5 et RCP 8.5.

La méthode du Delta consiste à déterminer un changement (ou une anomalie) qui correspond à la différence des moyennes entre une simulation de climat futur moins la simulation de climat présent (Lenderink et al., 2007), en calculant simplement un changement moyen sur l'ensemble de la distribution des observations (Colin, 2010).

La méthode de correction quantile-quantile $(Q-Q)$, ou quantile mapping consiste à estimer la correction en fonction des quantiles de la distribution de probabilité de la variable en question (Déqué, 2007). Un quantile de la distribution simulée est remplacé par le même quantile de la distribution observée.

Une fois estimée, la fonction de correction est appliquée à la variable issue des simulations de climat futur qui peut être finalement prise en compte dans des études d'impacts socioéconomiques dans le contexte des changements globaux.

\section{Résultats et discussions}

\section{1 Évaluation des modèles climatiques régionaux au cours de la période de contrôle 1981-2005}

De manière générale, la représentation du cycle saisonnier des pluies observées et simulées au cours de la période de référence (1981-2005) montre que les deux modèles RCA4MPI-ESM-LR et RCA4-CNRM-CM5 ont du mal à reproduire les pluies mensuelles observées à l'échelle du bassin du côtier oranais (Fig. 2). Le biais estimé entre les pluies observées et simulées présente en moyenne une variation de $-6 \%$ à $-70 \%$ (Tableau 2), il dépasse souvent $-50 \%$ pendant les mois d'hiver. Ainsi, les modèles CNRM et MPI sous- 

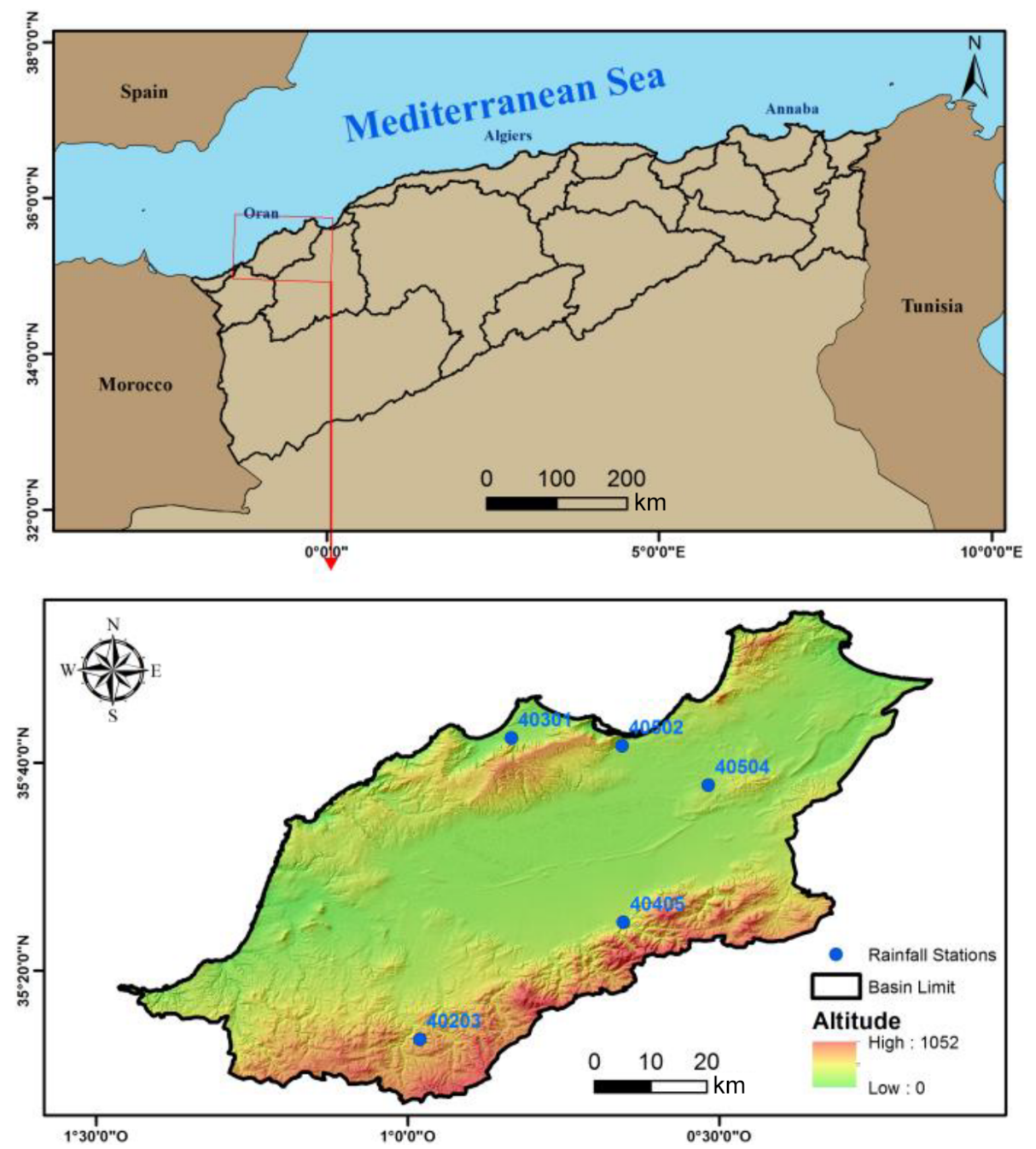

Figure 1. Localisation géographique des postes pluviométriques.

Tableau 1. Caractéristiques géographiques et statistiques des stations pluviométriques de la zone d'étude.

\begin{tabular}{llrrrr}
\hline Code & Nom stations & $X(\mathrm{~km})$ & $Y(\mathrm{~km})$ & $Z(\mathrm{~m})$ & P. Moy $(\mathrm{mm})$ \\
\hline 040203 & Oued berkeche & 165.1 & 220.2 & 450 & 352,69 \\
040301 & Bousfer & 180.4 & 273.3 & 84 & 317,05 \\
040405 & Tamzourah & 195.5 & 239.9 & 189 & 331,73 \\
040502 & Oran pépinière & 196.4 & 271.3 & 82 & 344,51 \\
040504 & El braya & 208.7 & 263.9 & 110 & 248,86 \\
\hline
\end{tabular}

estiment fortement les pluies à l'échelle du bassin du Côtier oranais en période humide, alors que le biais estimé en période sèche montre une surestimation des pluies. Les faibles quantités de pluies enregistrées en cette période sèche font que les biais relatifs estimés sont plus élevés.

Compte tenu de ces résultats, une correction du biais est primordiale afin de corriger les données brutes simulées par les modèles climatiques régionaux et améliorer la qualité des simulations futures.

\subsection{Correction de biais et projections futures}

Afin d'analyser l'évolution future des pluies après application des méthodes de correction de biais, un coefficient d'échange est estimé entre les pluies simulées futures corrigées et les pluies observées au niveau de chaque station (Fig. 3). L'analyse de la variabilité future des pluies concerne uniquement la période humide de la zone d'étude (OctobreMars) d'autant plus que les quantités de pluies les plus im- 

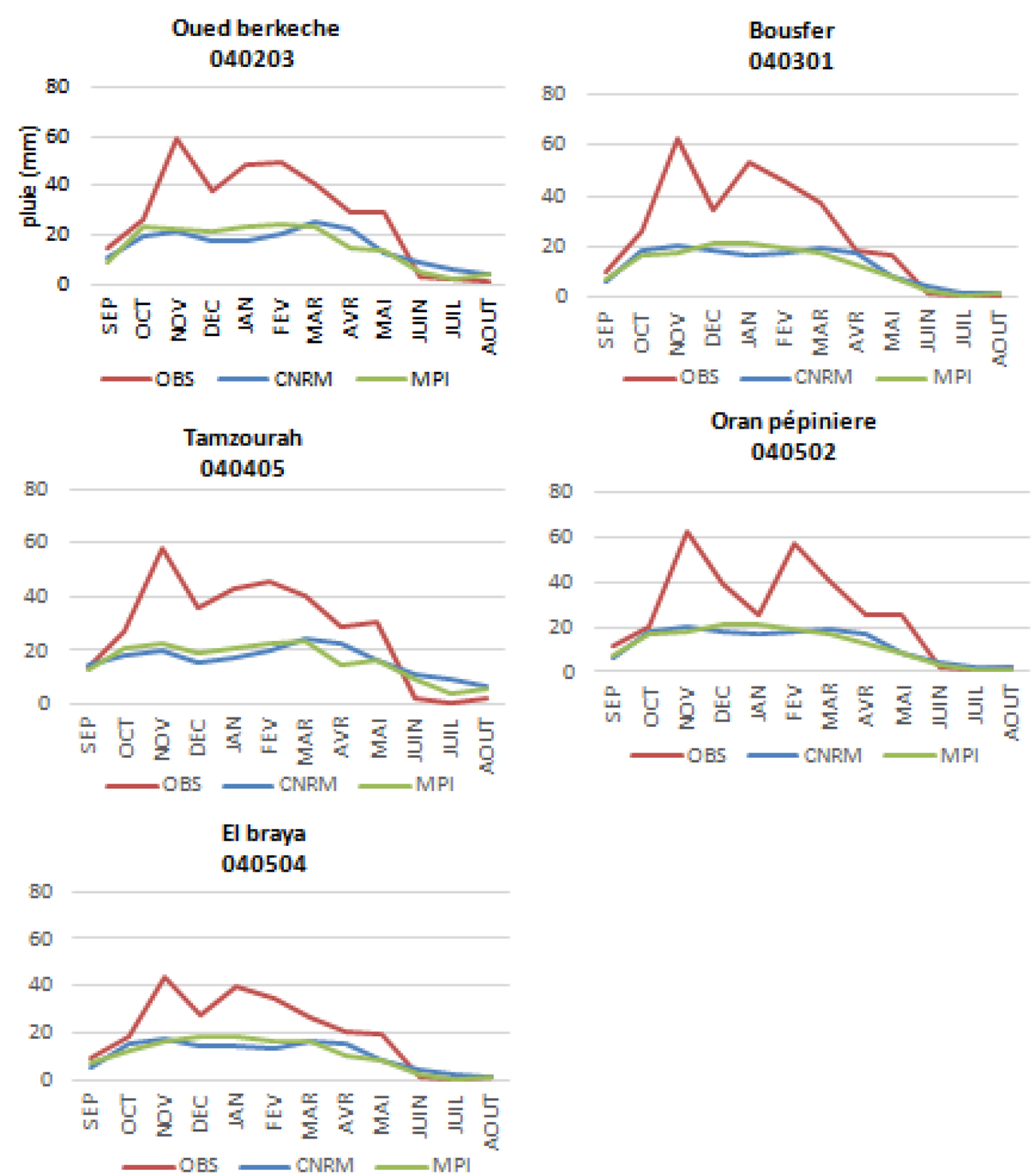

Figure 2. Représentation du cycle saisonnier par les MCR pour chaque station.

portantes qui participent aux écoulements sont enregistrées au cours de cette période (Fig. 2).

De manière générale, les variations des pluies simulées par le modèle MPI pour le scénario climatique RCP 4.5 au cours de la période 2075-2099 sont pratiquement similaires pour les deux méthodes de corrections et montrent une diminution de la pluviométrie comprise entre $-19 \%$ et $-38 \%$ à l'échelle du bassin oranais. Pour le RCP 8.5, les deux méthodes de correction montrent des changements différents des pluies pour l'ensemble des stations qui oscillent entre $-27 \%$ et $-47 \%$ pour la méthode Delta et entre $-41 \%$ et $-65 \%$ pour la méthode $Q-Q$.

Les simulations du modèle CNRM, sous le scénario RCP 4.5 mettent en évidence une diminution de $-7 \%$ à $-61 \%$ estimée par la méthode Delta à l'exception de la station 040502 (Oran pépinière) qui montre une augmentation de $+34 \%$. Les simulations issues de la méthode Q$\mathrm{Q}$ montrent une diminution des pluies de $-25 \%$ à $-41 \%$ exceptée pour les stations 040502 et 040504 qui indiquent respectivement une augmentation de $+68 \%$ et $+48 \%$ des pluies d'ici la fin du 21 ème siècle. Pour le RCP 8.5 , les projections futures corrigées par la méthode Delta révèlent une réduction des pluies de $-31 \%$ à $-47 \%$, alors que la méthode $Q-Q$ simule une réduction de $-34 \%$ à $-59 \%$ des pluies d'ici la fin du 21 ème siècle.

L'ensemble de ces résultats témoignent de la vulnérabilité du bassin côtier oranais à la variabilité climatique. Ainsi, ils permettent d'appréhender, de manière globale, non seulement les conséquences sur la disponibilité des ressources en eau mais aussi sur l'ensemble des activités tributaires à l'eau et à enjeu socio-économique telles que : l'agriculture, l'industrie, la santé et le tourisme. Néanmoins, pour une meilleure appréciation des impacts socio-économiques, une analyse plus approfondie en considérant plusieurs modèles climatiques et paramètres climatiques, est recommandée. 
Tableau 2. Biais estimé entre les pluies observées et simulées au cours de la période de référence 1981-2005.

\begin{tabular}{|c|c|c|c|c|c|c|c|c|c|c|c|c|}
\hline MCR & Sept. & Oct. & Nov. & Déc. & Jan. & Fév. & Mars & Avril & Mai & Juin & Juil. & Aout \\
\hline \multicolumn{13}{|c|}{ Oued berkeche (040203) } \\
\hline CNRM & $-27 \%$ & $-24 \%$ & $-64 \%$ & $-53 \%$ & $-63 \%$ & $-58 \%$ & $-39 \%$ & $-21 \%$ & $-55 \%$ & $219 \%$ & $204 \%$ & $171 \%$ \\
\hline MPI & $-38 \%$ & $-12 \%$ & $-62 \%$ & $-42 \%$ & $-53 \%$ & $-52 \%$ & $-42 \%$ & $-50 \%$ & $-53 \%$ & $93 \%$ & $11 \%$ & $-38 \%$ \\
\hline \multicolumn{13}{|c|}{ Bousfer (040301) } \\
\hline CNRM & $28 \%$ & $39 \%$ & $-36 \%$ & $3 \%$ & $-37 \%$ & $-23 \%$ & $4 \%$ & $80 \%$ & $4 \%$ & $446 \%$ & $430 \%$ & $825 \%$ \\
\hline MPI & $37 \%$ & $28 \%$ & $-44 \%$ & $21 \%$ & $-20 \%$ & $-17 \%$ & $-7 \%$ & $36 \%$ & $3 \%$ & $230 \%$ & $119 \%$ & $37 \%$ \\
\hline \multicolumn{13}{|c|}{ Tamzourah (040405) } \\
\hline CNRM & $11 \%$ & $-32 \%$ & $-65 \%$ & $-58 \%$ & $-59 \%$ & $-57 \%$ & $-41 \%$ & $-22 \%$ & $-46 \%$ & $332 \%$ & $857 \%$ & $245 \%$ \\
\hline MPI & $-6 \%$ & $-24 \%$ & $-61 \%$ & $-46 \%$ & $-52 \%$ & $-52 \%$ & $-41 \%$ & $-50 \%$ & $-47 \%$ & $257 \%$ & $358 \%$ & $-6 \%$ \\
\hline \multicolumn{13}{|c|}{ Oran pépinière (040502) } \\
\hline CNRM & $-42 \%$ & $-8 \%$ & $-67 \%$ & $-53 \%$ & $-32 \%$ & $-69 \%$ & $-51 \%$ & $-33 \%$ & $-66 \%$ & $89 \%$ & $8 \%$ & $-25 \%$ \\
\hline MPI & $-37 \%$ & $-16 \%$ & $-71 \%$ & $-46 \%$ & $-52 \%$ & $-66 \%$ & $-56 \%$ & $-49 \%$ & $-66 \%$ & $14 \%$ & $-56 \%$ & $-37 \%$ \\
\hline \multicolumn{13}{|c|}{ El braya (040504) } \\
\hline CNRM & $-38 \%$ & $-21 \%$ & $-60 \%$ & $-49 \%$ & $-64 \%$ & $-59 \%$ & $-37 \%$ & $-24 \%$ & $-58 \%$ & $252 \%$ & $1065 \%$ & $99 \%$ \\
\hline MPI & $-23 \%$ & $-33 \%$ & $-63 \%$ & $-34 \%$ & $-53 \%$ & $-53 \%$ & $-40 \%$ & $-47 \%$ & $-57 \%$ & $137 \%$ & $282 \%$ & $-23 \%$ \\
\hline
\end{tabular}

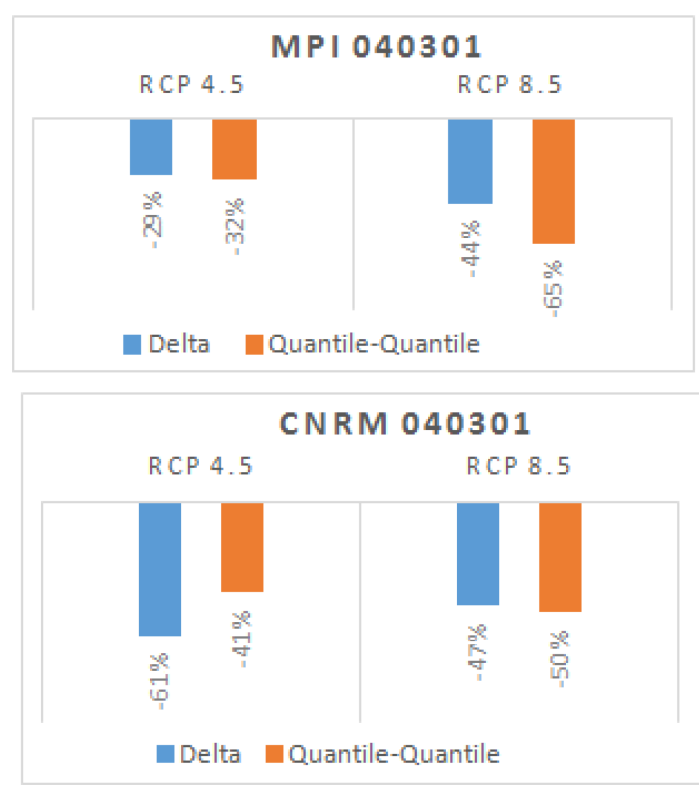

Figure 3. Variation des pluies futures (2075-2999) par rapport aux observations (1981-2005) après correction de biais par la méthode Delta et $Q-Q$ (cas de la station Bousfer 040301).

\section{Conclusions}

Au terme de cette recherche, il convient de retenir que d'une manière générale, les résultats de simulations ont mis en évidence une réduction de $-15 \%$ à $-40 \%$ des pluies à l'exception de la station côtière d'Oran pépinière qui montre une augmentation d'environ $34 \%$ des pluies à l'horizon 2099. Fort des résultats obtenus, cette recherche est une contribution pour la mise en place d'un outil d'aide à la planification et l'élaboration d'un plan d'actions opérationnel pour la gestion durable des ressources en eau dans le secteur d'étude. Toutefois, pour proposer les mesures d'adaptations les plus adéquates possibles, il est nécessaire de tenir compte des points suivants; (i) des erreurs liées aux modèles climatiques régionaux et aux méthodes de corrections de biais utilisées; (ii) des paramètres climatiques susceptibles d'affecter sensiblement l'hydrologie du bassin versant et avoir des répercussions sur les activités agricoles à savoir; l'évapotranspiration ainsi que l'intensité et la fréquence des pluies.

Disponibilité du code. Le calcul est basé sur les packages du langage R, qmap, dont les informations supplémentaires sont disponibles dans Gudmundsson (2012) et https://cran.r-project.org/web/ packages/qmap/index.html, la date du dernier accès : 19 avril 2020.

Disponibilité des données. Les données in-situ sont fournies par l'Agence Nationale des Ressources Hydraulique (ANRH). Les données projetées des modèles RCA4-MPI-ESM-LR et RCA4CNRM-CM5 sont fournies par l'étude coordonnée de recherche sur le climat à échelle régionale sur l'Afrique (CORDEX-Africa) https://esg-dn1.nsc.liu.se/search/cordex/, la date du dernier accès : 4 avril 2020) (SMHI, 2020).

Collaborateurs. ST, AZ et NM ont conceptualisé le travail; ST et $\mathrm{AZ}$ ont élaboré la méthodologie. AZ a fourni les simulations des 
modèles climatiques avec et sans correction des biais, et a supervisé le travail. NM a participé au traitement et à l'analyse des données. ST a fait l'analyse des données; a rédigé le manuscrit préliminaire; a fait la révision et l'édition de l'article. AZ a participé à la révision et à l'édition de l'article. la version finale de l'article a été approuvé par l'ensemble des auteurs.

Intérêts concurrents. Les auteurs déclarent qu'ils n'ont aucun conflit d'intérêts.

Clause de non-responsabilité. Publisher's note : Copernicus Publications remains neutral with regard to jurisdictional claims in published maps and institutional affiliations.

Déclaration du numéro spécial. This article is part of the special issue "Hydrology of Large River Basins of Africa". It is a result of the 4th International Conference on the "Hydrology of the Great Rivers of Africa", Cotonou, Benin, 13-20 November 2021.

Remerciements. Les auteurs souhaitent exprimer leur gratitude envers l'Agence Nationale des Ressources Hydrauliques (ANRH) pour avoir fourni les données pluviométriques sur lesquelles sont basées les analyses de cette étude. Nos remerciements sont adressés au Groupe de travail sur le climat régional du Programme mondial de recherche sur le climat, le CORDEX-Afrique (Coordinated Regional climate Downscaling Experiment), initiative du projet CMIP5 pour le Programme mondial de recherche sur le climat. Nous remercions également le Rossby Center pour la production et la mise à disposition de leur modèle atmosphérique régional, sortie RCA4. Enfin, nous tenons à remercions les reviewers pour leurs commentaires constructifs qui ont permis d'améliorer le manuscrit.

\section{Références}

ANRH : Impact des changements climatiques sur les ressources en eaux. Note de synthèse, 12p, 2007.

Bekkoussa, B., Meddi, M., and Jourde, H. : Forçage climatique et anthropique sur la ressource en eau souterraine d'une région semi-aride : cas de la plaine de Ghriss (Nord-Ouest algérien), Sécheresse 18, 173-184, 2008.

Colin, J., Déqué, M., Radu, R., and Somot, S. : Sensitivity study of heavy precipitation in Limited Area Model climate simulations : influence of the size of the domain and the use of the spectral nudging technique, Tellus A, 62, 591-604, 2010.

Déqué, M. : Frequency of precipitation and temperature extremes over France in an anthropogenic scenario : Model results and statistical correction according to observed values, Glob. Plane. Change, 57, 16-26, 2007.

Dobler, A. and Ahrens B. : Precipitation by a regional climate model and bias correction in Europe and South Asia, Meteorol. Z., 17, 499-509, https://doi.org/10.1127/0941-2948/2008/0306, 2008.
Fang, G. H., Yang, J., Chen, Y. N., and Zammit, C. : Comparing bias correction methods in downscaling meteorological variables for a hydrologic impact study in an arid area in China, Hydrol. Earth Syst. Sci., 19, 2547-2559, https://doi.org/10.5194/hess-19-25472015, 2015

GIEC : Changements climatiques 2014 : Rapport de synthèse, Contribution des Groupes de travail I, II et III au cinquième Rapport d'évaluation du Groupe d'experts intergouvernemental sur l'évolution du climat, Sous la direction de l'équipe de rédaction principale, édité par : Pachauri, R. K. et Meyer, L. A., GIEC, Genève, Suisse, p. 161, 2014.

Gudmundsson, L. : qmap : statistical transformations for postprocessing climate model output, $\mathrm{R}$ package version 1.0-3 [code], available at : http://cran.r-project.org/web/packages/qmap/ (last access : 19 April 2020), 2012.

Lenderink, G., Buishand, A., and van Deursen, W. : Estimates of future discharges of the river Rhine using two scenario methodologies : direct versus delta approach, Hydrol. Earth Syst. Sci., 11, 1145-1159, https://doi.org/10.5194/hess-11-1145-2007, 2007.

Ly, M., Segnon, A.C., D’haen, S., Totin, E., Noblet, M., Camara, I., and Pfleiderer, P. : Comprendre et interpréter les sorties de modèles climatiques pour la conduite des études de vulnérabilité : Guide à l'attention des praticiens, Climate Analytics gGmbH, Berlin, 21 pp., 2019.

Meddi, M., Assani, A. A., and Meddi, H. : Temporal variability of annual rainfall in the Macta and Tafna catchments, Northwestern Algeria, Water Resour. Manag., 24, 3817-3833, 2010.

Mendez, M., Maathuis, B., Hein-Griggs D., and Alvarado-Gamboa, L.F. : Performance Evaluation of Bias Correction Methods for Climate Change Monthly Precipitation Projections over Costa Rica, Water, 12, p. 482, https://doi.org/10.3390/w12020482, 2020.

SMHI (Swedish Meteorological and Hydrological Institute) : ESGF@LiU/CORDEX [data set], available at : https://esg-dn1. nsc.liu.se/search/cordex/, last access : 4 April 2020.

Taibi, S., Meddi, M., Souag, D., and Mahe, G. : Évolution et régionalisation des précipitationspluies au nord de l'Algérie (19362009), Climate and land surface changes in hydrology, IAHS Publ, 359, 191-197, 2013.

Teutschbein, C. and Seibert, J. : Bias correction of regional climate model simulations for hydrological climate-change impact studies : Review and evaluation of different methods, J. Hydrol., 456, 12-29, 2012.

Tong, Y., Gao, X., Han, Z., Xu, Y., Xu, Y., and Giorgi, F. : Bias correction of temperature and precipitation over China for RCM simulations using the QM and QDM methods, Clim. Dynam., 57, 1425-1443, https://doi.org/10.1007/s00382-020-05447-4, 2020.

Zeroual, A., Assani, A. A., and Meddi, M. : Combined analysis of temperature and rainfall variability as they relate to climate indices in Northern Algeria over the 1972-2013 period, Hydrol. Res., 48, 584-595, 2017.

Zeroual, A., Assani, A. A., Meddi, M., and Alkama, R. Assessment of climate change in Algeria from 1951 to 2098 using the Köppen-Geiger climate classification scheme, Clim. Dynam., 52, 227-243, 2019. 